\title{
PATTERNS AND KINETICS OF NEURITE EXTENSION FROM SYMPATHETIC NEURONS IN CULTURE ARE AGE DEPENDENT ${ }^{1}$
}

\author{
VINCENT ARGIRO*,2 AND MARY I. JOHNSON*, $^{*}$ \\ ${ }^{*}$ Department of Anatomy and Neurobiology and $\ddagger$ Department of Pediatrics, Washington University School of Medicine, St. \\ Louis, Missouri 63110
}

Received August 10, 1981; Revised November 2, 1981; Accepted November 24, 1981

\begin{abstract}
Long term (2- to 3-week) cultures of superior cervical ganglia (SCG) were established from rats and rat embryos ranging in age from 15 days of gestation (E15) to 279 days postnatal (P279). Cultures were grown on a collagen substratum and fed a serum-containing medium with added nerve growth factor. Radial outgrowth of neurites was measured as a function of time for up to 2 to 3 weeks. Computer-aided analysis generated estimates of onset time, initial rate, and subsequent changes in the rate of growth of these neurites. The explants from perinatal rats showed the fastest growth onset time ( 5 to $13 \mathrm{hr}$ ), fastest initial rate of growth $(370$ to $660 \mu \mathrm{m} / \mathrm{d}$ ), and a decline in growth rate during the first 2 weeks in culture. The outgrowth from these perinatal explants was composed of many small fascicles. Neurites from the prenatal explants (E15 to E20) began to grow within $22 \mathrm{hr}$ in vitro. Their rate of growth was lower initially ( 150 to $300 \mu \mathrm{m} / \mathrm{d}$ ) but increased to equal the perinatal explant initial rate before again falling to an intermediate level (200 to 300 $\mu \mathrm{m} / \mathrm{d})$. The outgrowth from prenatal explants contained fewer larger fascicles. Postnatal explants had low initial rates of growth (70 to $176 \mu \mathrm{m} / \mathrm{d}$ ) but exhibited an increasing growth rate in vitro, again approaching an intermediate rate of 200 to $250 \mu \mathrm{m} / \mathrm{d}$ after 2 to 3 weeks. Neurite outgrowth from the postnatal explants was delayed by an amount roughly correlated with the age of the animal from which they had come (up to $68 \mathrm{hr}$ for P49). The initial rate of growth also decreased with advancing postnatal age but reached an asymptote of about 50 to $150 \mu \mathrm{m} / \mathrm{d}$ at about P30. The outgrowth was initially sparse but became denser with time in culture. Thus, in a culture system in which medium composition and growth substratum are held constant, marked differences can be observed in pattern, latency, initial rate, and subsequent changes in rate of neurite extension among SCG explants from different ages of rats and rat embryos.
\end{abstract}

The growth of neurites from newly differentiating neurons as well as from regenerating proximal stumps has been of interest since the axon was recognized as a direct extension from the neuronal soma (Ramon y Cajal, 1917).

\footnotetext{
'We wish to thank Drs. Mary B. Bunge, Richard P. Bunge, and Patrick Wood for help and advice and Ms. Lisa Wartels and Mrs. Artree James for technical assistance. We are grateful for the use of computer facilities generously provided by Dr. Calton C. Hunt of the Department of Physiology and Biophysics and for the assistance and advice of Dr. Robert S. Wilkinson in the application of these facilities. Some of the analysis was completed with a microcomputer system funded in part by a grant from the Department of Pediatrics authorized by Dr. Philip R. Dodge. This work was supported by Grants NS14416 and NS15070 to M. B. Bunge from the National Institutes of Health. V. A. is supported on National Institutes of Health Training Grant GM07564.

${ }^{2}$ To whom correspondence should be addressed at Department of Anatomy and Neurobiology, Box 8108, Washington University School of Medicine, 660 South Euclid Avenue, St. Louis, MO 63110.
}

A number of the early investigations provided data on the rate of growth (Harrison, 1910; Ramon y Cajal, 1928; Speidel, 1933). Subsequent studies have provided growth rates for nerve fibers both in vivo (Gutmann, 1942) and in vitro. Bray (1973), for example, noted that superior cervical ganglion (SCG) neurons explanted from newborn rats extended neurites at the rate of 30 to $40 \mu \mathrm{m} / \mathrm{hr}$ on a collagen substratum during the initial days of culture. Many of these studies were short term and the growth rates were derived from observations over not more than several hours or days. In addition, few workers studied the rate of growth as a function of the age of the animal.

During studies on the development of neurotransmitter function in SCG neurons in culture, methods were developed to grow explants from rats of all ages in long term culture (Ross et al., 1977; Johnson et al., 1980). During this study, it was observed that, at a given time in culture, the length of neurites appeared to vary as a function of the age of the animal from which the neurons 
were taken. It also was noted that explants taken from older animals showed a delay in their onset of growth. Because of our interest in regeneration and the underlying basis of these age dependencies, we have pursued these observations. The studies reported here were aimed specifically at quantitating and describing the patterns of neurite extension from both embryonic and postnatal animals. A preliminary report of these findings has appeared in abstract form (Johnson and Argiro, 1979).

\section{Materials and Methods}

Culture procedures. SCG were dissected from rats and rat embryos ranging in age from 15 days of gestation (E15) to 279 days of postnatal life (P279). Rats of both sexes were used at random. The ages of all rats were determined on the basis of sperm-positive dates. The management of breeding was such that embryos were actually up to 0.5 day older than the ages cited. Embryos were removed from pregnant females under ether anesthesia. Postnatal animals were etherized and the SCG were removed under sterile conditions. The connective tissue capsule was removed from all ganglia except those taken from E18 or younger embryos. The capsule, if present on these ganglia, is too delicate to manipulate. The tissue removed from SCG of animals older than 10 to 14 days postnatal is not a true capsule but a connective tissue sheath common with the carotid and jugular vessels as well as the vagus nerve. Each ganglion was divided into explants of roughly equal size, 0.5 to $1.0 \mathrm{~mm}$ in diameter. Adult ganglia yielded 8 to 12 explants; E21 ganglia yielded 3 or 4 explants; E15 ganglia were left intact; intermediate ages were handled accordingly.

For all experiments reported here, an E21 ganglion explant was co-cultured with 1 or 2 explants from rats of different ages. The E21 explant thus was used to monitor the presence of uncontrolled variables from one experiment to the next, but no attempt was made to normalize the measurements to those from the E21 explants. The cultures were contained within unsealed all glass $35-\mathrm{mm}$ culture dishes (Bellco Glass, Inc., Vineland, NJ). Before plating the explants, the dish bottoms were coated with a double film of reconstituted rat tail collagen. The bottom layer was polymerized by exposure to ammonia vapors; the top layer, on which the explants were grown, was air dried, yielding a highly fibrous and long lasting growth substratum. The cultures were fed every 3 to 5 days with a medium containing $85 \%$ Eagle's minimum essential medium (Grand Island Biological Co.), 10\% human placental serum, $1 \%$ chick embryo extract, $0.6 \%$ glucose, $1 \%$ L-glutamine $(200 \mathrm{~mm})$, and 10 biological units/ml of nerve growth factor (NGF; Varon et al., 1972). The cultures were incubated at $35^{\circ} \mathrm{C}$ in a $5 \% \mathrm{CO}_{2}$ in air-humidified atmosphere.

Neurite measurement procedure. Neurite outgrowth from the SCG explants is visible as a radial halo with a distinct front or leading edge. Observations of living cultures at high magnification indicate that the outgrowth is quite synchronous and that the majority of growth cones are at the front. It is therefore possible to estimate the progress of neurite extension by monitoring the distance between this front and the explant border. The halo was visualized by low magnification ( $\times 10$ to 20 ) bright-field light microscopy. Initially, this image was recorded photographically and the positions of the explant border and growth cone front then were traced onto paper from the projected negative. In later experiments, the image of the living culture was projected directly onto paper, utilizing a low power inverted microscope designed for this purpose. The positions of the growth cone front for each explant were accumulated on a common sheet of paper as a series of irregularly shaped concentric rings (Fig. 1). Only sections of the halo edge unobscured by non-neuronal cell overgrowth and where the neurites were growing straight toward the periphery were traced. The measurements were taken at intervals of 1 to 3 days for the 1st week in vitro and at intervals of 3 to 5 days during the 2 nd and 3 rd weeks. The measurements were ended when the neurite front reached the edges of the dish, collided with the fronts of the other explants in the dish, or became unrecognizable amid the carpet of proliferating non-neuronal cclls.

Computer-aided data analysis. The paper tracings were digitized with the aid of a magnetostrictive graphic tablet (Houston Instruments, Inc., Austin, TX) interfaced to a TI-980B mini computer (Texas Instruments, Inc., Austin, TX). The validity of the digitizing process was checked by displaying the digital map of the tracing on a graphics cathode ray tube as each perimeter was entered. A sampling of the neurite lengths around the extent of the traced halo was obtained with a simple geometric algorithm: for each of approximately 128 points digitized on the growth cone front, the minimum distance to a point on the explant border was calculated. Thus, for each time point, a statistical population was obtained whose mean reflected the "neurite extent" or average radius of the growth cone front and whose standard deviation reflected the variability in the outgrowth from a single explant. These mean halo radii at each time point were obtained for each of the 4 to 9 explants of a given in vivo age surviving the experiment. Again, mean, standard deviation, and standard error of the mean (SEM) were calculated. For this secondary statistical population, the mean reflects the behavior of the neurites from all explants of a given age sample, and the standard deviation and SEM reflect variability from one culture to the next. It should be noted that these measurements are estimates of neurite lengths; it is the mean distance between the explant border and the growth cone front which is calculated.

The rate of neurite extension was calculated by two methods. The first method utilized the linear regression equations applied to overlapping sets of three time points to calculate growth rates throughout the experimental period. The time in vitro quoted for each rate so obtained is the mean of the three times of measurement. In this way, changes in the rate of neurite extension over the culture period could be studied. The variability in these rate measurements for a given population of explants was indexed by computing a $95 \%$ confidence interval (Remington and Schork, 1970).

In addition, a nonlinear regression analysis was performed on the neurite length data from the whole culturing period by an adaptation of the method of least squares (see Bennett, 1976). The equation fitted is:

$$
L=R_{0} / R_{1}\left(1-e^{-R_{1}\left(t-t_{0}\right)}\right)
$$




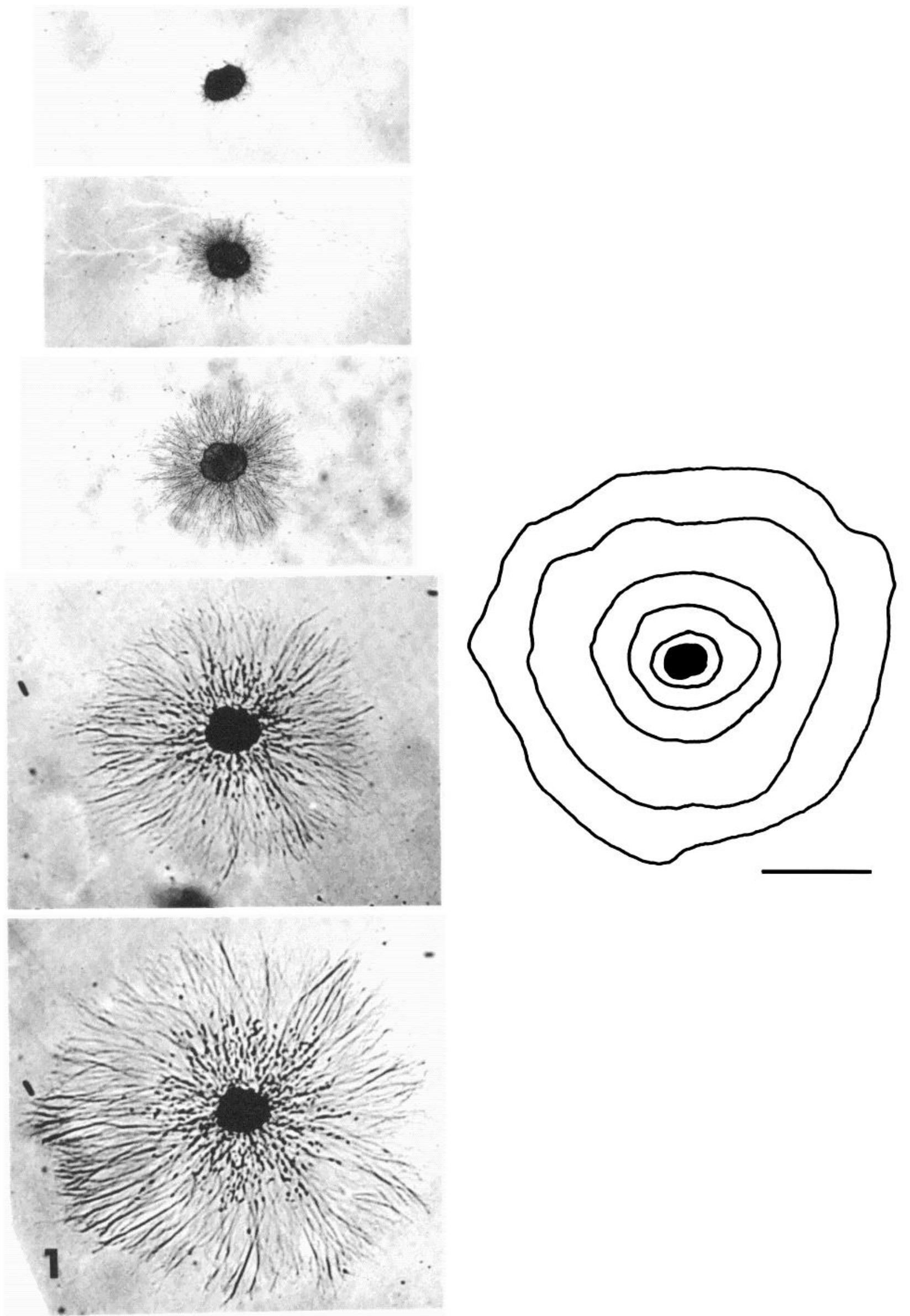

Figure 1. One superior cervical ganglion explant from an E21 rat embryo photographed in the living state at 1.2, 2.2, 2.8, 3.8, and 4.8 days in culture. On the right are the tracings of the neurite front at these times. Tracings such as these were used to determine neurite extension kinetics. Scale bar, $1 \mathrm{~mm}$. 
where $t$ is the time in vitro in days, $L$ is neurite length in micrometers, and the three adjustable parameters $t_{0}, R_{0}$ and $R_{1}$ are the onset time (days), initial growth rate (micrometers per day), and first order rate constant $\left(\right.$ days $\left.^{-1}\right)$, respectively. The above equation was chosen because changes in the rate of neurite outgrowth appeared to take an exponential form at least for the postnatal explants (see "Results") and particularly for the perinatal explants. The first derivative of the above equation, which gives the rate of growth as a function of time, is an exponential of the sign opposite to that of the original function. This procedure permitted a curvilinear extrapolation of the neurite growth function to the time of onset in lieu of the linear extrapolation obtained by the linear regression method. As the rate of neurite extension appears to change most rapidly during the initial period of growth, the values for initial growth rate obtained with the nonlinear fit may be better estimates of the actual values during the first hours of growth.

\section{Results}

General observations on patterns of growth. The data reported here derive from a series of eight experiments, including a total of approximately 600 SCG explants. Stereotyped patterns of neurite growth and behavior of accompanying non-neuronal cells (primarily Schwann cells and fibroblasts) were noted for embryonic, perinatal, and postnatal explants.

Embryonic explants began to manifest neurites within the 1st day of incubation. The initial outgrowth was weblike in appearance due to a reticular sheet of non-neuronal cells which were spread among and restricted to the area occupied by the neurite fascicles. The fascicles were very large (50 to $200 \mu \mathrm{m}$ ) in diameter (Fig. $2 a$ ) in

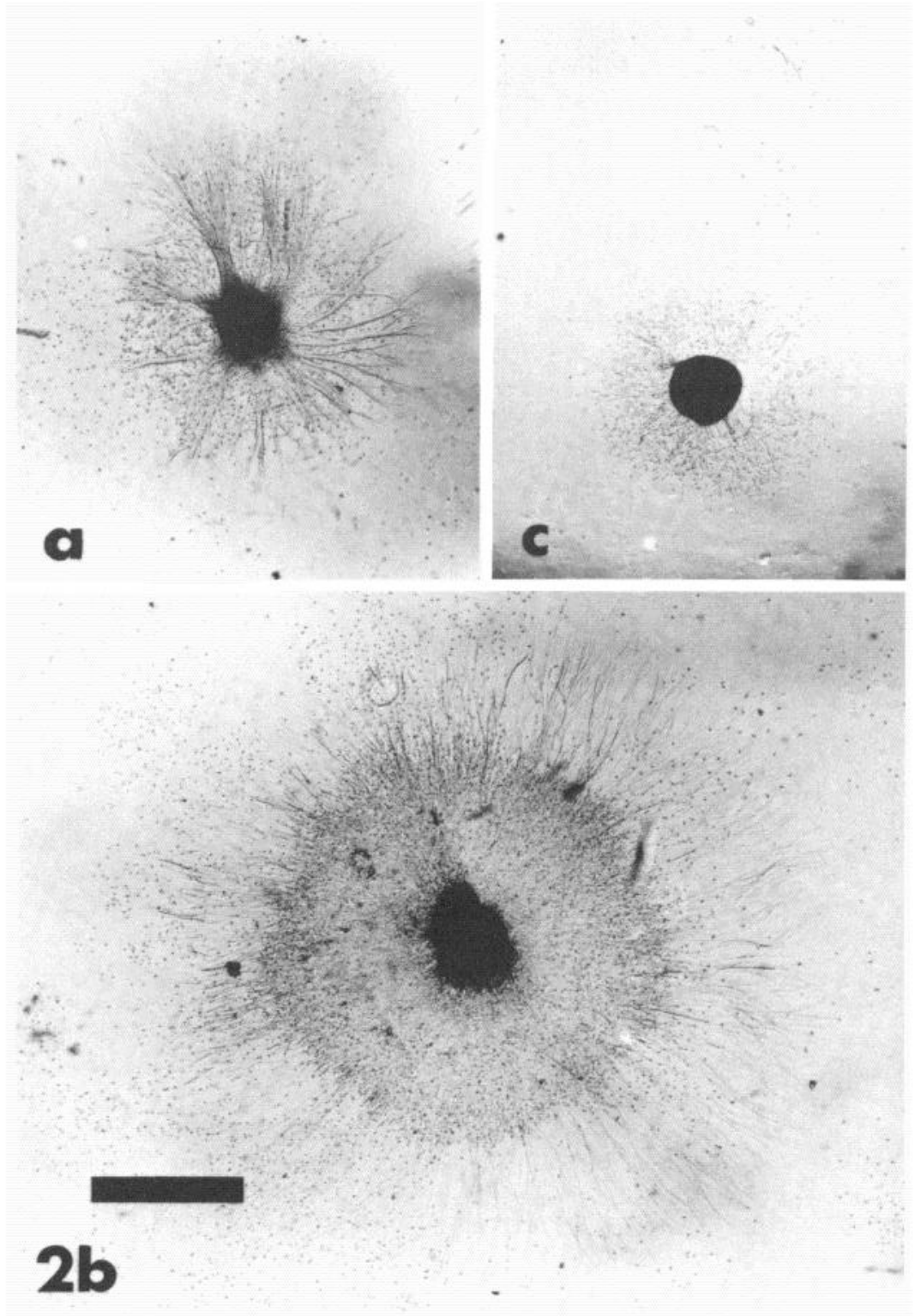

Figure 2. SCG explants after 6 days in culture. These three explants from E16 $(a)$, E21 $(b)$, and P49 $(c)$ rats were cultured in the same dish and photographed in the living state. Scale bar, $1.5 \mathrm{~mm}$. 
comparison to those extending from perinatal explants (Fig. $2 b$ ). In many cases, there were only 5 to 10 fascicles around the entire explant, compared with hundreds of small fascicles around a similarly sized perinatal explant. As the growth of these neurites accelerated (see below), they appeared to leave behind the halo of non-neuronal cells surrounding the explant and grow out onto the collagen substratum. Only later, during the 2nd or 3rd week in vitro, did a confluent monolayer of fibroblasts cover the dish. The embryonic explants themselves remained lucent throughout the culturing period, probably indicating a fairly high rate of cell survival and lack of necrosis.

Perinatal explants also produced a visible outgrowth within the 1st day in culture (see Fig. $2 b$ ). In this case, however, the outgrowth was composed of small anastomotic fascicles and was relatively free of non-neuronal cells. The front of growth cones remained well in advance of the front of migrating non-neuronal cells until the 2nd or 3rd week in culture when, again, a confluent carpet of Schwann cells and fibroblasts often developed. The perinatal explants generally remained lucent but more often than the embryonic explants developed a dark center probably indicative of necrotic cell loss at the core of the explant.

Explants from animals 1 week postnatal or older exhibited yet a third growth pattern (Fig. 2c). Initiation of neurite extension was delayed for several days (see below) and outward migration of a number of non-neuronal cells always preceded the appearance of extending neurites. Fascicles were moderate in diameter in comparison to the other two groups but, at least initially, were few in number. The density of the outgrowth appeared to increase during the culturing period and the fronts of growth cones and migrating non-neuronal cells advanced at about the same rate. These postnatal explants are more opaque when placed in culture but may become more so with time; thus, it is not clear to what extent this opacity is due to necrosis.

Neurite length as a function of time. The first stage in the quantitative analysis consisted of plotting the mean distance between the growth cone front and explant border as a function of time for each group of explants of a given age from each experiment. Such data from a representative experiment are plotted in Figure 3. Note that the neurite extension from the embryonic explants (E15 in this case) is first behind that from the perinatal (E21) group but becomes more rapid after several days. The pattern for the perinatal (E21) group is different. The neurite extension proceeds first very rapidly but gradually slows. The two older postnatal groups (P16 and P279) show a common pattern differing only quantitatively. Neurite outgrowth is slow at first, relative to the E21 group, but the curves turn upward with time in culture. The P2 explants show a pattern intermediate between the E21 and older postnatal groups. Note that the rate of growth early in culture and the total length that the neurites achieved are related directly to the in vivo age of the postnatal explants. These plots suggested to us three parameters whose age dependence we might specifically characterize: neurite growth onset time, initial rate of neurite growth, and changes in growth rate as a function of time in culture.

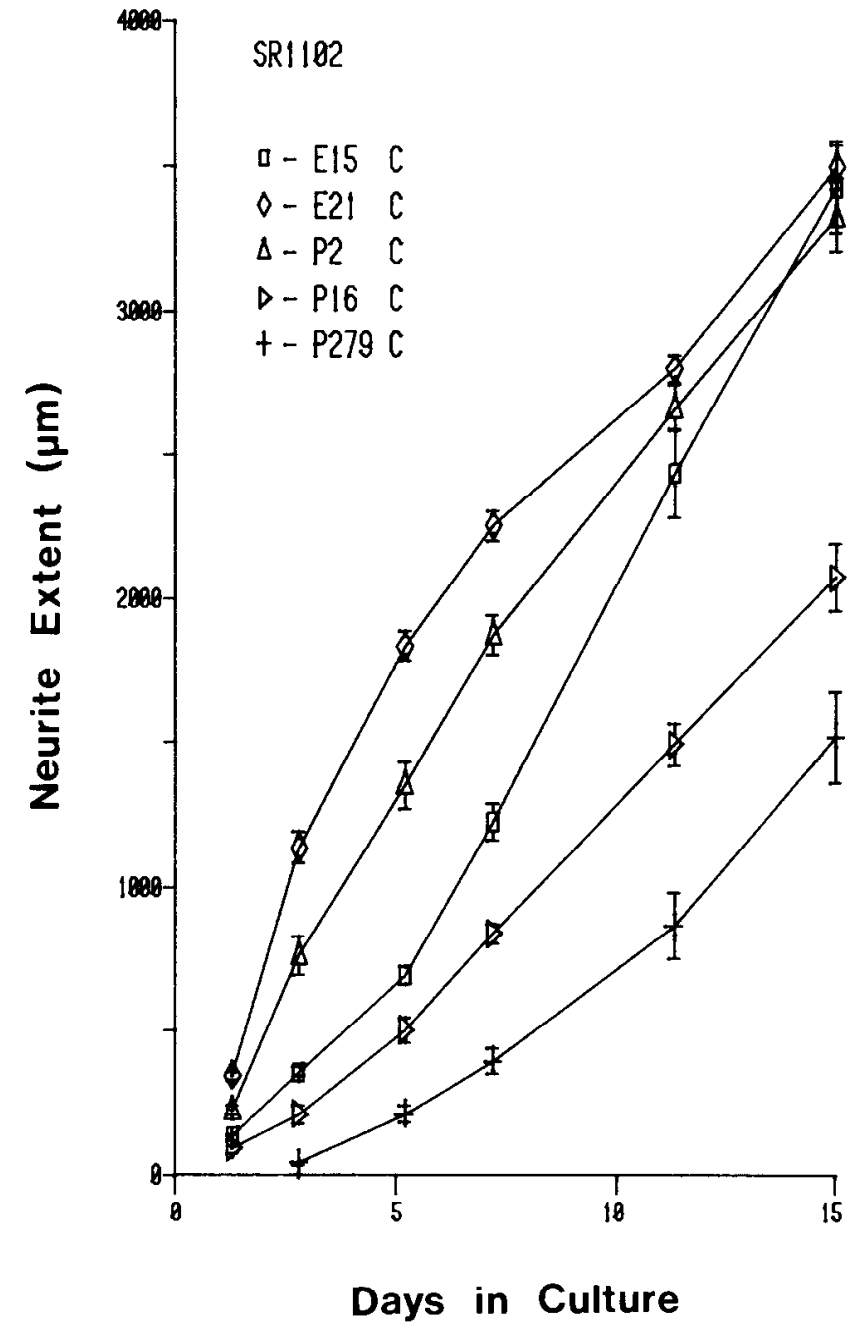

Figure 3. Neurite extent (distance between explant border and growth cone front) as a function of time in culture for five ages. Each point represents a mean for 4 to 9 explants. Error bars are for \pm SEM.

Neurite growth onset time. As it was not practical to observe all of the cultures directly during their initial period of incubation, we adopted a method for estimating the time of onset of neurite extension utilizing an extrapolation of the neurite length versus time data. We chose the nonlinear fit parameter $t_{0}$ for the estimate. In Figure 4 , this estimate of onsct time is plotted as a function of the age of the rat from which the explants were taken. Note the close agreement among the five values plotted for E21 explants. This agreement among values derived from experiments conducted over a period of more than a year is an index of the repeatability of the culture preparation. As indicated qualitatively above, the onset of neurite extension from embryonic and perinatal explants is prompt, occurring within 6 to $24 \mathrm{hr}$ of explantation. The onset of regeneration of postnatal explants, on the other hand, is delayed approximately 20 to $70 \mathrm{hr}$. There appears to be a weak correlation between postnatal age and the magnitude of this effect.

Initial rate of neurite extension. When the nonlinear fit parameter $R_{0}$, the estimate of the initial rate of neurite extension, is plotted as a function of in vivo age (Fig. 5), 


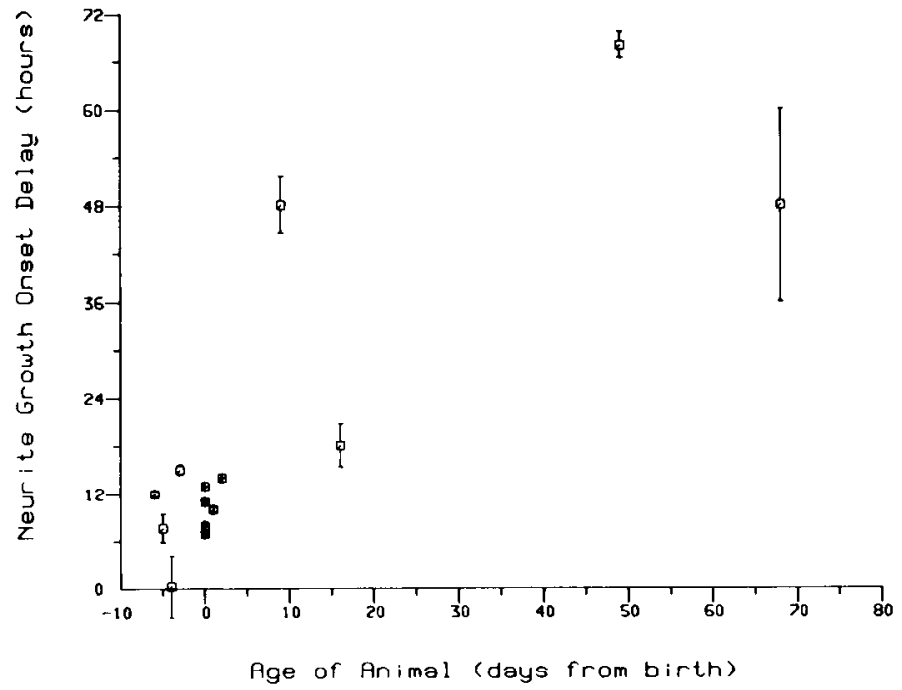

Figure 4. Delay before onset of neurite growth as a function of the age of the animal. The values were derived by nonlinear fit to the data for 4 to 9 explants in each case. Error bars are for \pm SEM.

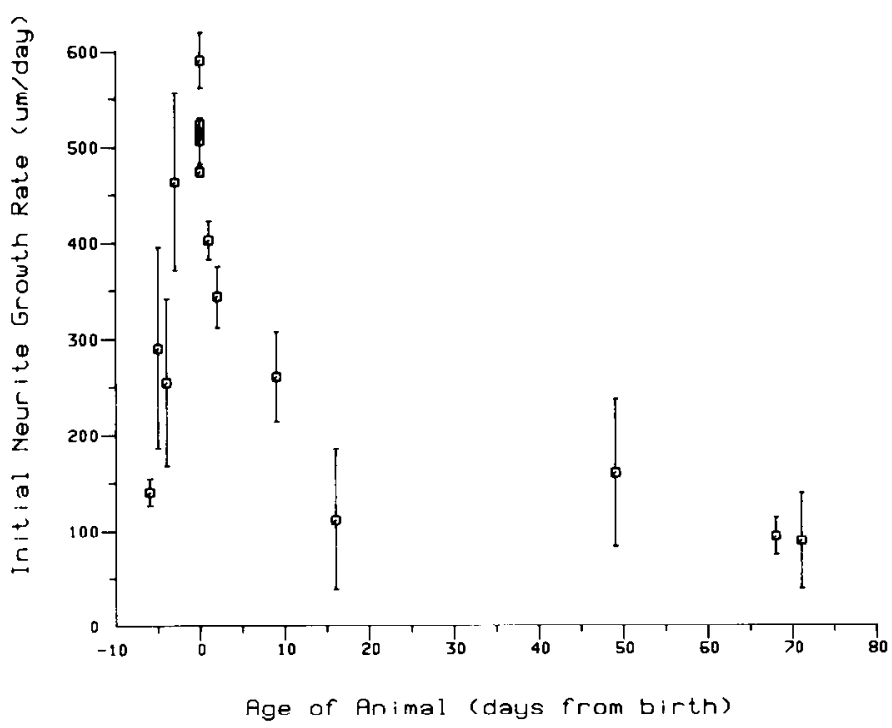

Figure 5. Initial neurite growth rate as a function of the age of the animal. The values were derived by nonlinear fit to the data for 4 to 9 explants in each case. Error bars are for \pm SEM.

a strikingly smooth function emerges. Again, an indication of the relative lack of systematic errors from one experiment to the next is provided by the agreement of values for E21 explants. All but one value is close to 500 $\mu \mathrm{m} / \mathrm{d}$. Note that these values are also the highest obtained for any age. Values obtained for the embryonic explants begin with a minimum of about $140 \mu \mathrm{m} / \mathrm{d}$ for E15 and tend to increase approximately linearly with advancing age toward the maximum at E21. The initial neurite extension rate for postnatal explants, on the other hand, declines with advancing age. The decline is very steep from E21 to P16 but becomes more gradual thereafter, appearing to approach an asymptote of about 100 $\mu \mathrm{m} / \mathrm{d}$ for explants from rats older than P30.
Changes in neurite growth rate in culture. It is evident from the data presented in Figure 3 that the neurite growth rates of all ages of explants do not remain constant during the culturing period but in fact change in a predictable manner. To study these effects in more detail, growth rates were computed by linear regression of the length versus time data throughout the culture period. A complete catalogue of these data is presented in Table I.

Examination of these data yielded a set of trends for the various stages of explants. These trends are, however, better indicated by plotting the neurite growth rate as a function of time in culture with the age of the animal as a parameter (Fig. 6). The growth rate of neurites from embryonic explants typically increases from a lower level $(150$ to $250 \mu \mathrm{m} / \mathrm{d}$ ) to a maximum (300 to $500 \mu \mathrm{m} / \mathrm{d}$ ) during the 1st week in culture before decreasing again (200 to $300 \mu \mathrm{m} / \mathrm{d})$ during the balance of the culture period. Perinatal explants (including P2 to P5) exhibit a progressive decline from an initial maximum (500 to 600 $\mu \mathrm{m} / \mathrm{d})$ to an apparent asymptote $(200$ to $300 \mu \mathrm{m} / \mathrm{d})$ by the 3rd week. Neurites from postnatal explants grow initially at their minimum rate $(50$ to $150 \mu \mathrm{m} / \mathrm{d})$ but increase their rate considerably (150 to $250 \mu \mathrm{m} / \mathrm{d})$ during the 1st week in culture. A decrease in the growth rate sometimes follows during the 2nd and 3rd weeks.

If, instead of following the changes occurring over time in culture for a given age group, we plot neurite growth rate as a function of the age of the animal, with time in culture as a parameter, we can note changes in age dependence for fixed times in culture (Fig. 7). We see, in this way, that the striking age dependence of initial neurite growth rate illustrated for the nonlinear fitting method in Figure 5 and for the earliest three-point linear regression in Figure 7 is greatly attenuated later in the culturing period. That is, regardless of the age of the tissue at the time of explantation, neurite extension is proceeding at a common rate of 150 to $250 \mu \mathrm{m} / \mathrm{d}$ by the 3rd week in culture.

\section{Discussion}

This study characterizes and quantitates the age dependency of several parameters of neurite growth in explant cultures of rat superior cervical ganglia. The onset of growth occurs within hours in embryonic and perinatal explants, while 1 to 4 days elapse before neurites emerge from postnatal explants. The initial rate of growth of the embryonic neurites is one-half to threequarters that of the perinatal neurites (see Table I; Fig. $5)$. The postnatal explants are not only delayed in onset of growth but initially grow at a markedly lower rate than either the embryonic or perinatal explants. Changes in growth rate over time in culture for a given explantation age were observed consistently. Growth rates of perinatal neurites decline rapidly from an initial maximum. The rates of neurite extension from both embryonic and adult explants accelerate during the 1st week in vitro but may decline thereafter. Therefore, at explantation, the perinatal neurons may be in a state more supportive of neurite extension than the neurons from older or younger neurons.

It is unlikely that the differences in initial growth rate can be explained by less optimal conditions for health and survival in the explants from the embryonic or adult 
TABLE I

Summary of neurite growth rates

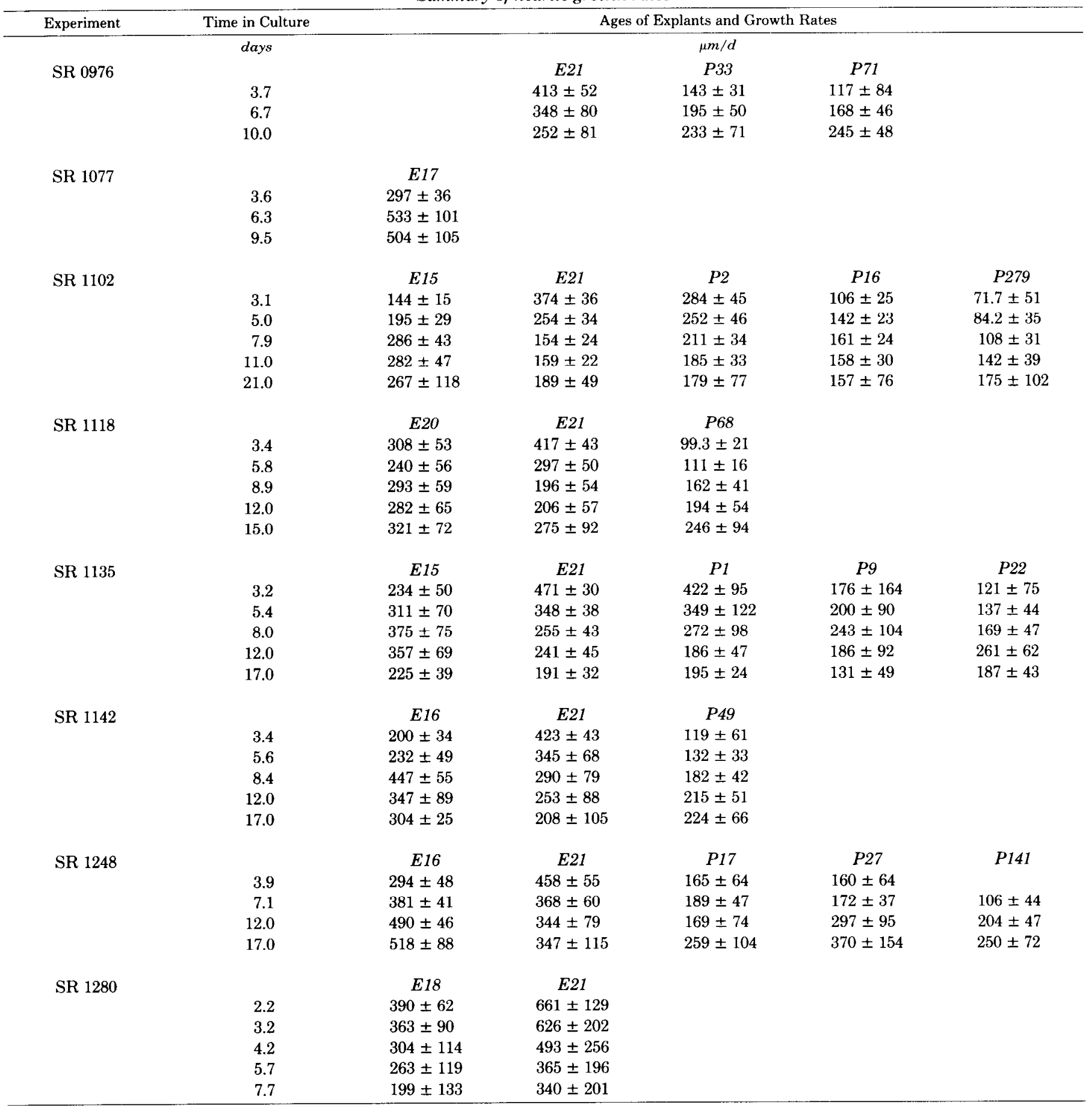

"Values represent the mean $\pm 95 \%$ confidence interval.

animals. Embryonic explants tended to flatten onto the substratum and appeared as healthy or healthier than the thicker explant of the perinatal explants. Prior studies have shown that after 4 to 5 weeks in culture, the percentage of cell survival in adult explants equals that of perinatal explants (Johnson et al., 1980). The adult explants, despite the initial delay and initial low rate of growth, have a vigorous neuritic halo by several weeks in culture and continue to grow for months.

Many factors, undoubtedly inter-related, are impor- tant for nerve fiber growth (for recent reviews, see Bunge et al., 1982; Letourneau, 1982). We will consider several in interpreting our present results.

Differences in growth cone motility and cell surface properties probably play an important role in the observed differences in neurite growth. Interaction of the growth cone with its substratum (collagen, extracellular matrix, other neurites, or non-neuronal cells) may either promote or inhibit its advancement, depending on the adhesiveness of the contact (Letourneau, 1975a, b; 


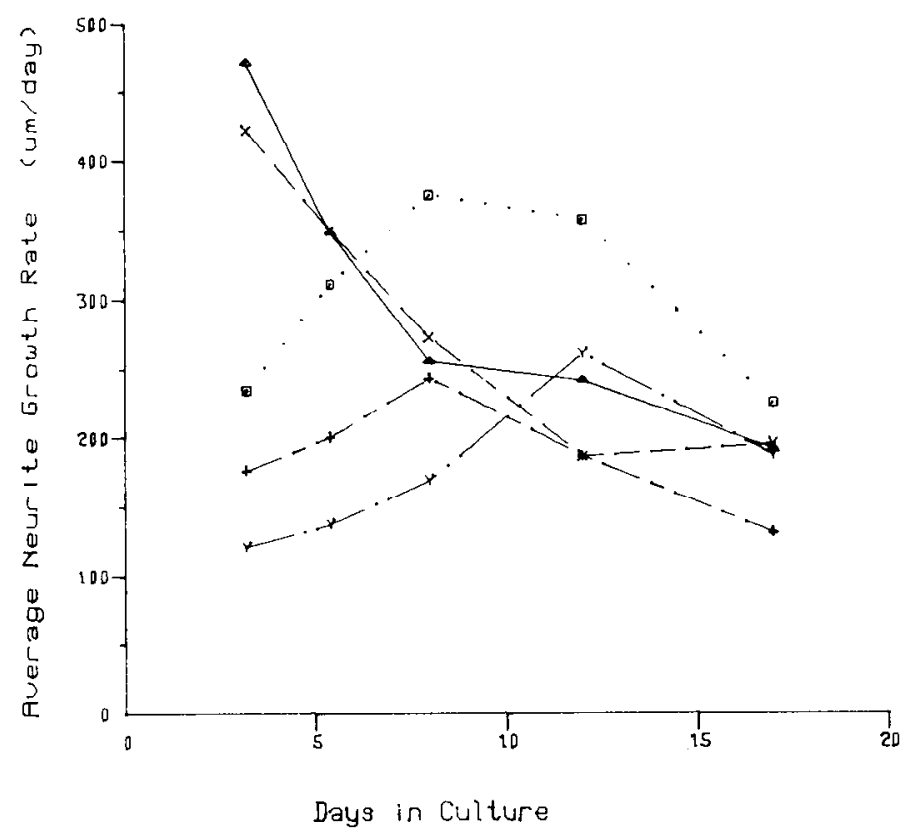

Figure 6. Neurite growth rate as function of time in culture for five ages of explant in a single experiment (SR 1135). The ages shown are: E15 $(\square \ldots . \square)$, E21 $(\triangle-\triangle)$, P1 $(\times---\times)$, P9 (+...+), and P22 (Y.....Y)

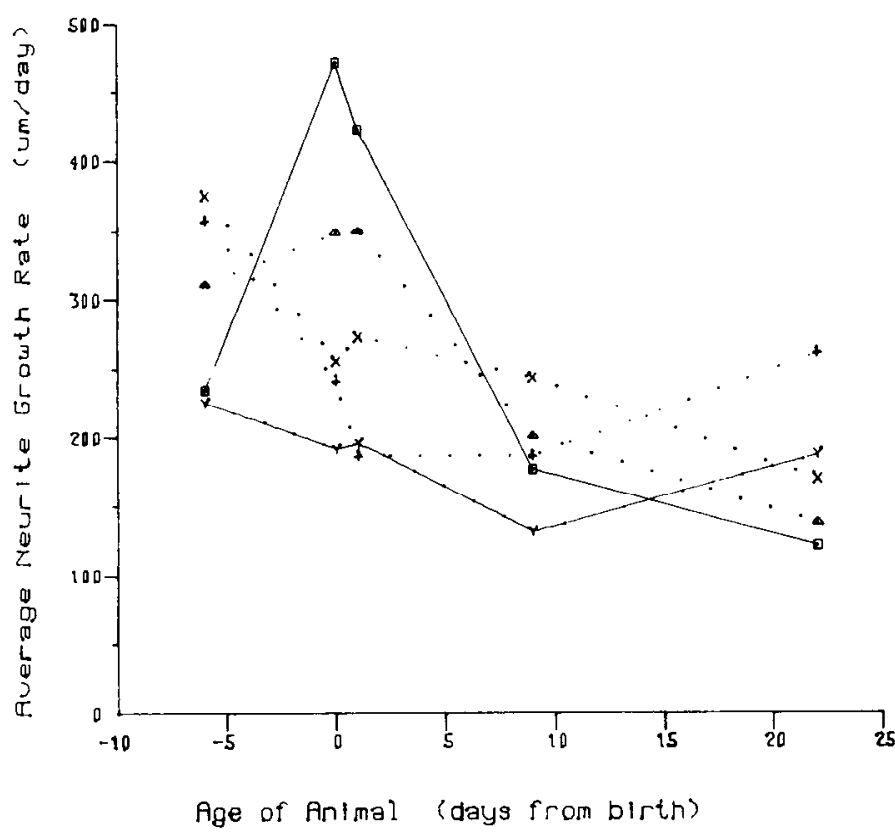

Figure 7. Neurite growth rate as a function of the age of the animal for five times in culture in a single experiment (SR 1135). The times shown are $3.2(\square \square \square), 5.1(\triangle \cdots . \triangle), 8.0$ $(\times \cdots \cdots \times), 12(+\cdots \cdots+)$, and $17 \mathrm{~d}(\mathrm{Y}-\mathrm{Y})$. Note the marked age dependence of the neurite growth rate at $3.2 \mathrm{~d}$ and its almost complete disappearance by $17 \mathrm{~d}$ (solid lines).

Schubert and Whitlock, 1977). Direct light microscopic observation and quantitative time lapse analysis of growth cones from explants and dissociated neurons are currently in progress in our laboratory (Argiro et al., 1981). Whether individual growth cones move steadily along the substratum or whether their translation is wandering or interrupted by instances of detachment and retraction could explain the differences in the long term rate of advance of a front of growth cones measured in this study. In addition, we hope to gain information on the form, size, and kinetics of generation and collapse of the lamellipodial and filopodial extensions (Chen, 1981).

We have observed a tendency for the neurites of cells of embryonic origin to remain confined initially to the region of outwardly migrating non-neuronal cells. Their initial growth rate therefore may be limited by the outward migration of non-neuronal cells and their apparent preferential adhesion to such cells. However, after several days in culture, at the same time that their growth rate is increasing, they leave the non-neuronal cell carpet behind and grow onto the collagen substratum. Early in culture, the membrane properties of the neurites and/or non-neuronal cells from E15 to E17 explants may be such that the neurites prefer the non-neuronal cellular substratum. With time in culture, surface properties may change such that the neurites now prefer the collagen substratum and grow rapidly upon it. The striking fasciculation of the neurites from embryonic explants also may offer an explanation for their slower initial growth. Rutishauser and Edelman (1980) recently have demonstrated that extensive fasciculation tends to slow linear growth in cultures of chick spinal ganglia. Fasciculation is related to the presence of "cell adhesion molecule" (CAM) on the neurite surface. Antibodies to CAM reduce fasciculation and promote the linear growth of neurites. The interactions among non-neuronal cell migration, substrata, and fasciculation are being studied further in our laboratory (Roufa et al., 1981). The presence of nonneuronal cells cannot totally explain differences in the pattern and kinetics of growth. When the growth of nonneuronal cells is prevented using an antimitotic agent (fluorodeoxyuridine), the age differences remain. Because these results are complicated by a direct effect of the drug on neurite extension, these results will be published separately. A preliminary report has appeared (Argiro and Johnson, 1981).

The metabolic status and response to axotomy of the SCG neurons from rats of different ages are likely to be different. Many cells in the embryonic (E15 to E19) SCG are still dividing at the time of explantation (Hendry, 1977). Growth from neurons "born" in vitro is in fact initial growth and not regeneration. In contrast, the neurons of the perinatal explants were actively growing at explantation and therefore are regenerating in the culture setting in response to NGF. The adult neurite is regenerated from a mature neuron which, for some weeks or months, was in contact with its target and now has undergone a chromatolytic response. Evidence that these cells may in fact be in different states of metabolic activity or gene expression is provided by previous work in our laboratory which demonstrated that the accrual of cholinergic properties by these neurons is also age dependent (Ross et al., 1977). The function relating the activity of the enzyme choline acetyltransferase after 1 month in culture to the age of the animal at the time of SCG explantation (Fig. 2 of Ross et al., 1977) is strikingly similar to the function relating the initial neurite growth rate and the age of the animal (Fig. 5).

Recent studies by Skene and Willard (1981a, b) have 
shown that several specific proteins are synthesized in the soma and axonally transported in regenerating neuronal systems but are absent in non-regenerating systems. They hypothesize that these "growth-associated proteins" (GAPs) are critically involved in axonal growth. The perinatal neuron may be fully equipped in this regard and therefore grow well at the time of explantation. The embryonic neuron may not yet have begun to express the genes for these GAPs; the adult neurons may require the period of chromatolysis to reactivate them. It is known that peripheral neurons show increases in RNA and protein synthesis during chromatolysis (see Veraa et al., 1979, for review); expression of growth-essential genes and their products may be involved.

The SCG neuron is known to be dependent on NGF for survival and maintenance during development (LeviMontalcini and Angeletti, 1963; Chun and Patterson, 1977) and the adult neuron is also responsive to NGF (Levi-Montalcini and Booker, 1960; Thoenen et al., 1971; Gorin and Johnson, 1980). Studies in the chick have shown a correlation between the presence of cell surface receptors for NGF and NGF responsiveness (Herrup and Shooter, 1975; Godfrey and Shooter, 1979). It is therefore possible that the slower initial growth rate of the embryonic neurites is due to a lower density of NGF receptors and lower responsiveness to NGF. The observed increase in the growth rate might be due in part to an increase in responsiveness to NGF.

An in vivo study by Black and Lasek (1979) has provided evidence that the rate of nerve fiber regeneration in the sciatic nerve of the rat is age dependent. The age range studied was 3 to 4 weeks to 6 to 9 months postnatal. The measured rates, ranging from 4.7 to $2.4 \mathrm{~mm} / \mathrm{d}$, manifested the same decline in growth rate with advancing age seen for postnatal neurons in culture in our study. The rates are all several times greater than those seen in culture, but this is consistent with many previous culture and in vivo studies and probably represents a systematic deficiency in the culture environment. The artificial substratum is certainly a candidate for this deficiency. The advantages of the present study over in vivo approaches include the capability of studying neurons at much earlier stages of development and of continuous monitoring of the growth from a given explant over many days. Further application of these techniques should provide information on the inter- and intracellular mechanisms which govern the extension of neuronal processes during development and regeneration.

\section{References}

Argiro, V., and M. Johnson (1981) Inhibition of neurite extension by fluorodeoxyuridine is age-dependent, dose-dependent and reversible. Soc. Neurosci. Abstr. 7: 346.

Argiro, V., M. B. Bunge, and M. Johnson (1981) Quantitative time-lapse analysis of growth cone motility of cultured rat autonomic neurons. J. Cell Biol. 91: 92a.

Bennett, W. R., Jr. (1976) Scientific and Engineering Problem Solving with the Computer, pp. 321-330, Prentice-Hall, Englewood Cliffs, NJ.

Black, M. M., and R. J. Lasek (1979) Slowing of the rate of axonal regeneration during growth and maturation. Exp. Neurol. 63: 108-119.

Bray, D. (1973) Branching patterns of isolated sympathetic neurons. J. Cell Biol. 56: 702-712.
Bunge, M. B., M. I. Johnson, and V. Argiro (1982) Studies of regenerating nerve fibers and growth cones. In Spinal Cord Reconstruction, C. Kao and R. P. Bunge, eds., Raven Press, New York, in press.

Chen, W. -T. (1981) Mechanisms of retraction of the trailing edge during fibroblast movement. J. Cell Biol. 90: 187-200.

Chun, L. L. Y., and P. H. Pattersun (1977) Role of nerve growth factor in the development of rat sympathetic neurons in vitro. I. Survival, growth, and differentiation of catecholamine production. J. Cell Biol. 75: 694-704.

Godfrey, E. W., and E. M. Shooter (1979) Nerve growth factor receptors on chick sympathetic ganglion cells. Soc. Neurosci. Abstr. 5: 767.

Gorin, P., and E. M. Johnson (1980) Effects of long-term nerve growth factor deprivation on the nervous system of the adult rat: An experimental autoimmune approach. Brain Res. 203: 27-42.

Gutmann, E. (1942) Factors affecting recovery of motor function after nerve lesions. J. Neurol. Neurosurg. Psychiatry 5: 81-95.

Harrison, R. G. (1910) The outgrowth of the nerve fiber as a mode of protoplasmic movement. J. Exp. Zool. 9: 787-846.

Hendry, I. A. (1977) Cell division in the developing sympathetic nervous system. J. Neurocytol. 6: 299-309.

Herrup, K., and E. M. Shooter (1975) Properties of $\beta$-nerve growth factor receptor in development. J. Cell Biol. 67: 118125.

Johnson, M., and V. Argiro (1979) Comparison of growth patterns of cultured sympathetic neurons from perinatal and postnatal rats. Soc. Neurosci. Abstr. 5: 679.

Johnson, M. I., C. D. Ross, and R. P. Bunge (1980) Morphological and biochemical studies on the development of cholinergic properties in cultured sympathetic neurons. II. Dependence on postnatal age. J. Cell Biol. 84: 692-704.

Letourneau, P. C. (1975a) Possible roles for cell-to-substratum adhesion in neuronal morphogenesis. Dev. Biol. 44: 77-91.

Letourneau, P. C. (1975b) Cell-to-substratum adhesion and guidance of axonal elongation. Dev. Biol. 44: 92-101.

Letourneau, P. C. (1982) Nerve fiber growth and its regulation by extrinsic factors. In Neuronal Development, N. C. Spitzer, ed., Plenum, New York, in press.

Levi-Montalcini, R., and P. U. Angeletti (1963) Essential role of the nerve growth factor in the survival and maintenance of dissociated sensory and sympathetic embryonic nerve cells in vitro. Dev. Biol. 7: 653-659.

Levi-Montalcini, R., and B. Booker (1960) Excessive growth of the sympathetic ganglia evoked by a protein isolated from mouse salivary glands. Proc. Natl. Acad. Sci. U. S. A. 46: 373-384.

Ramon y Cajal, S. (1917) Recollections of my life (H. Craigie, translator). In Memoirs of the American Philosophical Society Vol. 8, pp. 368-369, American Philosophical Society, Philadelphia.

Ramon y Cajal, S. (1928) Degeneration and Regeneration of the Nervous System, Vol. 1, p. 229, Hafner, New York.

Remington, R. D., and M. A. Schork (1970) Statistics with Applications to the Biological and Health Sciences, pp. 253281, Prentice-Hall, Englewood Cliffs, NJ.

Ross, D., M. Johnson, and R. Bunge (1977) Development of cholinergic characteristics in adrenergic neurones is age dependent. Nature 267: 536-539.

Roufa, D., M. Johnson, and M. B. Bunge (1981) Differences in neuritic growth pattern depending upon neuritic age and culture substrata. J. Cell Biol. 91: 91a.

Rutishauser, U., and G. M. Edelman (1980) Effects of fasciculation on the outgrowth of neurites from spinal ganglia in culture. J. Cell Biol. 87: 370-378.

Schubert, D., and C. Witlock (1977) Alteration of cellular adhesion by nerve growth factor. Proc. Natl. Acad. Sci. 
U. S. A. 9: 4055-4058.

Skene, J. H. P., and M. Willard (1981a) Changes in axonally transported proteins during axon regeneration in toad retinal ganglion cells. J. Cell Biol. 89: 86-95.

Skene, J. H. P., and M. Willard (1981b) Axonally transported proteins associated with axon growth in rabbit central and peripheral nervous systems. J. Cell Biol. 89: 96-103.

Speidel, C. C. (1933) Studies of living nerves. II. Activities of ameboid growth cones, sheath cells, and myelin segments, as revealed by prolonged observation of individual nerve fibers in frog tadpoles. Am. J. Anat. 52: 1.
Thoenen, H., P. U. Angeletti, R. Levi-Montalcini, and R. Kettler (1971) Selective induction by nerve growth factor of tyrosine hydroxylase and dopamine $\beta$-hydroxylase in the rat superior cervical ganglion. Proc. Natl. Acad. Sci. U. S. A. 68: 1598-1602.

Varon, S. J., J. Nomura, J. Perez-Polo, and E. Shooter (1972) Isolation and assay of the nerve growth factor proteins. In Methods of Neurochemistry, R. Fried, ed., Vol. 3, pp. 203229, Marcel Dekker, Inc., New York.

Veraa, R. P., B. Grafstein, and R. A. Ross (1979) Cellular mechanisms in axonal growth. Exp. Neurol. 64: 649-698. 